\title{
Urgences
}

\section{L'allusion métaphorique comme support de l'interprétation}

\section{Francine Belle-Isle}

Numéro 30, décembre 1990

L'autre du texte

URI : https://id.erudit.org/iderudit/025625ar

DOI : https://doi.org/10.7202/025625ar

Aller au sommaire du numéro

Éditeur(s)

Urgences

ISSN

0226-9554 (imprimé)

1927-3924 (numérique)

Découvrir la revue

Citer cet article

Belle-Isle, F. (1990). L'allusion métaphorique comme support de

l'interprétation. Urgences, (30), 47-57. https://doi.org/10.7202/025625ar d'utilisation que vous pouvez consulter en ligne.

https://apropos.erudit.org/fr/usagers/politique-dutilisation/ 


\title{
L'allusion métaphorique comme support de l'interprétation
}

\author{
Francine Belle-Isle
}

\begin{abstract}
Dans ce qui se présente à moi comme espace de la lumière, ce qui est regard est toujours quelque jeu de la lumière et de l'opacité. C'est toujours ce miroitement qui était là tout à l'heure au cœur de ma petite histoire, c'est toujours ce qui me retient, en chaque point, d'être écran, de faire apparaître la lumière comme chatoiement, qui le déborde. Pour tout dire, le point de regard participe toujours de l'ambiguitté du joyau.

Jacques Lacan, Les quatre concepts fondamentaux de la psychanalyse, p. 89-90.
\end{abstract}

Lire un texte pour qui s'intéresse à l'inconscient, c'est le prendre à la lettre. À la lettre de la métaphore, cette nomination perverse du monde, griffure d'un réel qui s'absente dans le temps même de son évocation. À la lettre de la représentation, quand s'est calmée l'obsession de la capture du sens dans la fascination que crée la danse des signifiants. C'est tenir compte à chaque fois de la possible déportation du signifié, de son exil dans le terrain vague du langage, sous la barre du refoulement, juste à la barre, là où dans une condensation obscure persiste et insiste un interdit en continuel déplacement.

Quand mes étudiants en psychanalyse littéraire, pleins de bonne volonté mais trop préoccupés de décider du référent une fois pour toutes, avant même que d'entendre ce que les mots du texte ont à dire, s'énervent et, au bord de l'exaspération, me lancent cet ultimatum: "Mais enfin, de quoi s'agitil ici?", je les renvoie volontiers à ce passage des Confessions ${ }^{1}$ qui raconte les tentatives exhibitionnistes de JeanJacques Rousseau et je les invite à faire avec moi un petit exercice de lecture...

1 Jean-Jacques Rousseau, Les confessions, 3, t. I, p. 88-90. Dans cet article, les références au texte de Rousseau renvoient aux CEuvres complètes, coll. “Bibliothèque de la Pléiade ", Paris, Gallimard, 1959-1969, 4 vol. parus. 


\section{Un homme montre ses fesses 2}

Délicieusement partagé entre la honte et le plaisir, un homme montre... ses fesses aux passantes dans de petites rues mal éclairées. En apparence, quoi de plus banal dans l'insolite! Avec en plus quelque chose de puéril, qui rend la scène un peu navrante. Et pourtant, on devine sans mal qu'il y a là un effet de sens dont la portée dépasse certainement le simple geste exhibitionniste. L'aveu est tellement chargé d'affects, il arrive dans la trame narrative d'une façon si déroutante, si pressante, qu'il trahit l'urgence de son surgissement. Le lecteur vient tout juste d'apprendre, avec beaucoup d'émotion, les terribles conséquences d'une bizarre affaire autour d'un petit ruban, et il espère un certain répit; au contraire on le presse d'écouter encore une histoire pas très transparente, comme si, d'avoir été libérée une fois, la parole n'arrivait plus à s'endiguer:

J'allois chercher des allées sombres, des réduits cachés où je pusse m'exposer de loin aux personnes du sexe dans l'état où j'aurois voulu pouvoir être auprès d'elles. Ce qu'elles voyoient n'étoit pas l'objet obscène, je n'y songeois même pas, c'étoit l'objet ridicule; le sot plaisir que j'avois de l'étaler à leurs yeux ne peut se décrire. II n'y avoit delà plus qu'un pas à faire pour sentir le traitement désiré, et je ne doute pas que quelque résolue en passant ne m'en eut donné l'amusement, si j'eusse eu l'audace d'attendre. ${ }^{3}$

Quand Starobinski insiste pour situer ce récit dans un champ beaucoup plus complexe que ne le suggère Rousseau

2 Ce que Jean-Jacques exhibe ici, c'est son derrière et non son sexe. Du moins c'est ce que semblent croire, et sans l'ombre d'un doute - et pourquoi pas? après tout, puisque ça n'a ici vraiment pas d'importance - Pierre-Paul Clément et tous les autres critiques avant lui: "Ce que les exhibitionnistes ont coutume d'offrir au regard d'autrui, c'est "l'objet obscène", c'est-à-dire le sexe. Lui, pour solliciter l'attention des Gabrielle ou des Goton de ses réves, présente "l'objet ridicule", autrement dit cette partie charnue de son anatomie sur laquelle s'était abattue la main sévère de $M^{\text {lie }}$ Lambercier" (Jean-Jacques Rousseau. De l'éros coupable à l'éros glorieux. Neuchâtel, La Baconnière, 1976. p. 107). Je pense que l'équation est un peu trop simple. Que les fesses scient la partie * ridicule" de l'anatomie, qu'elles excitent le rire plus naturellement que ne le peut le sexe, je le veux bien. Mais parce que le sexe est facilement un *objet obscène", qu'il ne puisse pas devenir éventuellement, dans les replis d'un psychisme, un * objet ridicule " et alors cesser d'être un * objet obscène ", voilà ce qui me paraît moins sûr.

3 Jean-Jacques Rousseau, op. cit., p. 88-89. 
lui-même, quand il répète sa conviction d'une vérité qui déborde largement les lieux communs de l'exhibitionnisme ${ }^{4}$, il sait bien qu'ici il ne s'agit pas du simple plaisir de se montrer. Ce qu'il ne dit pas, c'est qu'il s'agit, au moins tout autant, du plaisir de se cacher. Du plaisir qu'il y a à se cacher lorsque précisément on accepte de montrer sa nudité... On conviendra que Jean-Jacques prend bien des précautions pour ne pas montrer ce qu'il montre: il recherche des endroits sombres, des coins retirés, le fond d'une cour où il peut compter se réfugier facilement, des caves « longues et obscures». Il s'offre aux regards, oui, mais " de loin ", et à la sauvette, sans même attendre de goûter au plaisir d'être vu. Il renonce si vite au bénéfice de son geste qu'on se demande pourquoi il l'a osé. Il faut donc admettre qu'il retire peut-être quelque profit de sa dérobade, dès que s'est manifesté son désir de se montrer.

Et puis, montre-t-il vraiment ce qu'il a envie de montrer? Au bout du compte, si c'était son sexe en érection qu'il aurait aimé montrer, qu'il montre malgré tout, d'une manière détournée, avec des raffinements pervers, c'est-à-dire *l'état où [il] aurai[t] voulu pouvoir être" auprès des femmes? Ce n'est pas impensable. Il suffit de poser certaines équivalences métaphoriques. Quand Jean-Jacques montre ses fesses, c'est comme s'il montrait son pénis. Ce n'est pas sans raison qu'il choisit des femmes comme partenaires de son acte; il indique ainsi clairement le caractère hétérosexuel de l'échange et marque sa tentative de séduction du signe de la différence:

Dans cette confiance j'offrois aux filles qui venoient au puits un spectacle plus risible que séducteur; les plus sages feignirent de ne rien voir, d'autres se mirent à rire, d'autres se crurent insultées et firent du bruit. 5

Il faudrait tout souligner dans ces deux phrases. JeanJacques résume ici une théorie du regard et c'est bien l'incroyable sinuosité de l'espace scopique qui est identifiée et mise à profit. Quelque chose de "plus risible que séducteur* est donné à voir d'abord (et avec réticence encore, ce qui explique d'ailleurs que * les plus sages feignirent de ne rien voir *), donné comme un point d'ancrage, comme une référence immédiate. À supposer que l'œil se contente sobrement de cet

4 Jean Starobinski, Jean-Jacques Rousseau. La transparence et l'obstack, suivi de Sept essais sur Rousseau, coll. « Tel ", Paris, Gallimard, 1976, p. 207.

5 Jean-Jacques Rousseau, op. cit., p. 89. 
objet précis, Jean-Jacques s'attend à enregistrer une réaction de moquerie et c'est pourquoi « d'autres se mirent à rire *. Mais ce n'est manifestement pas ce qu'il souhaite ultimement, parce que dans ce cas l'histoire finirait ici. Heureusement pour lui, certaines femmes laissent circuler plus librement leur regard, lui permettent de déborder vers la zone périphérique ${ }^{6}$. L'effet produit n'est plus alors de l'ordre du rire, mais de l'ordre du scandale. C'est la visée séductrice qui l'emporte finalement, malgré l'écart dans le dérisoire. Il est évident, en effet, que ces regards de femmes, précisément parce qu'ils voient au-delà, plus avant, autre chose, sont les seuls nécessaires pour que soit satisfait le désir. Que cette * autre* chose ne se laisse voir que parce qu'elle reste cachée, c'est une affaire entendue, mais cela ne change rien ${ }^{7}$. Jean-Jacques compte absolument sur ces prolongements indiscrets du regard féminin pour poser, dans une reconnaissance implicite, une virilité masquée. C'est là que se trouve, inavouée et inavouable, sa prime de plaisir.

\section{Soudain une voix d'homme}

Mais cette gageure repose sur une mise fragile. Elle n'a de poids que dans l'instantanéité, que dans l'extrême fugacité de l'acte. La fin de l'histoire ne permet aucun doute là-dessus. Elle lève toute ambiguïté, brusquement. L'équilibre est rompu: le spectre de la castration, la peur qu'il suscite, le bouleversement qu'il provoque, tout devient clair:

J'entends une voix d'homme sur laquelle je n'avois pas compté et qui m'allarma: je m'enfonçai dans les souterrains au risque de m'y perdre; le bruit, les voix, la voix d'homme me suivoient toujours; j'avois compté sur l'obscurité, je vis de

\footnotetext{
6 Chez Rousseau, le regard est toujours un regard avide, extraordinairement désireux de ce qu'il voit, comme affamé par une vérité impossible à trouver. C'est aussi souvent un regard qui se voile, par affolement devant l'objet, à cause d'une panique incontrôlable devant la révélation d'une réalité trop brutale. C'est un regard qui palpite, coincé qu'il est entre l'exigence de son désir et l'impact heurtant du réel.

7 Jacque Lacan résume bien la dialectique du regard pervers, cette incursion de l'œil vers un ailleurs caché: "Ce qui compte, ce n'est pas que l'autre voit [sic] où je suis, c'est qu'il voit [sic] où je vais, c'est-à-dire, très exactement, qu'il voit [sic] où je ne suis pas. Dans toute analyse de la relation intersubjective, l'essentiel n'est pas ce qui est là, ce qui est vu. Ce qui la structure, c'est ce qui n'est pas là " Le séminaire, Les écrits techniques de Freud. coll. a Le champ freudien ", Paris, Seuil, 1975, p. 249).
} 
la lumière. Je frémis; je m'enfonçai davantage; un mur m'arrêta, et ne pouvant aller plus loin il fallut attendre là ma destinée. En un moment je fus atteint et saisi par un grand homme portant une grande moustache, un grand chapeau, un grand sabre, escorté de quatre ou cinq vieilles femmes, armées chacune d'un manche à balai, parmi lesquelles j'appercus la petite coquine qui m'avait décelé, et qui voulait sans doute $\mathrm{me}$ voir au visage. ${ }^{8}$

Coup de théâtre. Rupture du rythme. Changement de décor. Cette * voix d'homme», qui se détache du fond sonore, brise l'artifice. Elle cristallise l'angoisse de Jean-Jacques, accélère sa fuite, donne un sens à son épouvante. La menace de la castration est là, en toute lumière, *un mur * infranchissable se dresse, il faut faire face. Jean-Jacques peut bien dire qu'il ne "comptait" pas sur la résonance de cette "voix d'homme*, mais doit-on le croire? En tout cas, l'homme au sabre n'est pas un personnage secondaire, il arrive juste à temps dans la narration pour relancer l'intérêt dramatique et assumer l'entière responsabilité du dénouement.

Tant qu'on pouvait jouer sur la présence du sexe dans son absence même, on pouvait maintenir une sorte de non-lieu par rapport à la castration. Mais quelle précarité dans la performance! Combien de temps peut-on faire comme si l'envers était l'endroit, comme si le manque était jouissance? Combien de temps peut-on être sûr que ce qui demeure invisible existe pour vrai, plus réellement encore que s'il était offert au regard? En mimant sur son corps propre les audaces folles du désaveu pervers, en retenant dans l'être ce qu'il écarte dans le paraître, Jean-Jacques repousse désespérément une échéance. Il reporte à plus tard, mais jamais assez tard, l'acceptation de l'écart, de la séparation, de la distance et de la perte. Il ne me viendrait pas à l'idée de contester les conclusions de Starobinski sur le scandale provoqué dans l'âme de Rousseau par la conscience de la perte de la transparence. Mais si Jean-Jacques a, plus qu'un autre, souffert du mensonge des apparences, il a aussi tenté d'en tirer parti, de faire servir la surface trouble au maintien de l'illusion ${ }^{9}$. Et s'il est

8 Jean-Jacques Rousseau, op. cit., p. 89.

9 C'est déjà toute la problématique du * voile" chez Rousseau qui est dessinée ici. Faut-il óter le voile ou le garder? Y a-t-il une réponse à cette question? La solution, ce serait peut-être de s'en tenir à l'interrogation, comme en suspens... 
juste de dire que * Rousseau découvre le paraître en victime du paraître * 10 , il apprend très vite à n'en être pas que malheureux. Puisque de toute façon * on ne saura [...] jamais bien à qui l'on a affaire ${ }^{11}$, il faut arracher à cette souffrance la condition de son salut. Même si ce salut reste provisoire, surtout s'il n'est qu'un répit.

\section{L'être/le paraître}

Dans la Quatrieme promenade, Rousseau s'interroge longuement à propos du mensonge. Qu'est-ce que mentir? Peuton arriver à distinguer des cas où l'on est justifié de taire la vérité ou de la déguiser? Il se lance alors dans « d'embarrassantes discussions ", où il n'est pas toujours facile de le suivre tellement sa pensée se perd en nuances, et entreprend de reconsidérer les mérites de la transparence. Dans son argumentation, un point paraît faire la différence et esquisser déjà la manière perverse d'aborder la question du vrai et du faux. D'abord, dit-il, pour qu'une vérité soit due, il faut qu'elle soit ou puisse être utile, qu'elle intéresse la justice; autrement, on n'est pas obligé de la dire, et ce n'est pas mentir de la maquiller ou même de dire le contraire. Que toute vérité ne soit pas bonne à dire, cela va de soi, mais a-t-on le droit d'échanger le silence pour un mensonge, fût-il pieux? Rousseau pense que oui :

Je me souviens avoir lu dans un Livre de Philosophie que mentir c'est cacher une vérité que l'on doit manifester. II suit bien de cette définition que taire une vérité qu'on n'est pas obligé de dire n'est pas mentir; mais celui qui non content en pareil cas de ne pas dire la vérité dit le contraire, ment-il alors, ou ne ment-il pas? Selon la définition l'on ne sauroit dire qu'il ment; car s'il donne de la fausse monnoye à un homme auquel il ne doit rien, il trompe cet homme, sans doute, mais il ne le vole pas. ${ }^{12}$

Quelque chose gêne dans ce raisonnement. Les enchaînements sont trop rapides, trop chargés d'implications pour qu'on accepte la conclusion sans réticence. Bien sûr, il existe de bons mensonges, qui deviennent nécessaires quand le bien d'autrui

10 Jean Starobinski, op. cit., p. 20.

11 Jean-Jacques Rousseau, Discours sur les sciences et les arts, t. III, p. 8.

12 Jean-Jacques Rousseau, Quatrième promenade, t. I, p. 1026. 
les commande. Mais encore faut-il y être forcé par les circonstances, ne pas avoir le choix en quelque sorte. Rousseau va plus loin que cela. Ses paroles admettent la surenchère gratuite, la liberté entière de dire le contraire de ce qu'on peut ne pas dire. C'est ce saut délibéré dans une fausseté évitable qui crée le malaise et rend l'option plus ambiguë.

Mais Rousseau explique sa position en des termes qui nous permettent de mieux saisir les détours de sa démonstration:

Ne pas dire ce qui est vrai et dire ce qui est faux sont deux choses très différentes, mais dont peut neanmoins résulter le même effet; car ce résultat est assurement bien le même toutes les fois que cet effet est nul. Par tout où la vérité est indifferente l'erreur contraire est indifferente aussi; d'où il suit qu'en pareil cas celui qui trompe en disant le contraire de la vérité n'est pas plus injuste que celui qui trompe en ne la déclarant pas; car en fait de vérités inutiles, l'erreur n'a rien de pire que l'ignorance. Que je croye le sable qui est au fond de la mer blanc ou rouge, cela ne m'importe pas plus que d'ignorer de quelle couleur il est. ${ }^{13}$

Ce que Rousseau propose à notre entendement, c'est tout simplement l'abolition des contraires, chaque fois que les conséquences semblent indifférentes par rapport à la méconnaissance de la réalité. À partir du moment où l'on ne sait pas quelque chose, ce qui peut venir pallier cette absence ne change rien à la situation de base. Il y a comme une accoutumance à l'ignorance, que les modifications apportées par le vrai et le faux n'ébranlent pas vraiment. À la lecture de ces lignes, on jurerait que Rousseau raisonne ainsi parce qu'il a peur de la vérité! Il la noie dans les eaux troubles de l'ignorance et de l'erreur. Pour un peu, il irait jusqu'à dire qu'à défaut de rester dans l'ignorance, il vaut mieux être dans l'erreur que dans la vérité. Pire encore, à cause de la gratuité du «mensonge», il suggère presque de consolider l'ignorance par l'erreur, comme si deux précautions valaient mieux qu'une, quand il s'agit d'éloigner la vérité... Autrement dit, il est d'une sage prévoyance de construire tout de suite l'illusion qui servira de rempart contre la possible intuition de la vérité. Comme quoi chez Rousseau la prudence vient souvent obscurcir les voies du dévoilement et de la transparence. 
54

Dans ses efforts pour éloigner la réalité de la castration, le pervers a cette attitude face à l'ordre du vrai et du faux. Il en annule les effets par d'incroyables feintes, qui assurent le maintien de sa sécurité pulsionnelle. Sa sincérité, il la place dans une zone à part, où il est libre de dire et de ne pas dire, selon qu'il juge opportun ou non de soulever le voile sur une vérité qu'il nie, parce qu'il voudrait la savoir inutile et sans intérêt. Jean-Paul Valabrega fait remarquer le drôle de rapport que la perversion entretient avec la vérité:

Le problème est sans doute difficile parce qu'on a souvent l'impression que le pervers n'a pas accès au mensonge, c'est-à-dire aux catégories du vrai et du faux; qu'il est endeçà du mensonge [souligné dans le texte]. On pourrait aussi décrire les choses en disant que l'aveu et le désaveu sont, chez le pervers, également mensongers. ${ }^{14}$

En-deçà du mensonge, le pervers l'est certainement, puisque son discours ne se situe jamais dans l'explicite. Il navigue en des lieux d'ambiguité qui le ramènent toujours au bord d'un paradoxe. Quand il parle, il ne se soucie pas d'abord de dire vrai ou faux, il parle et il attend de voir ce que ca donne, comment ça résonne à ses oreilles comme à celles des autres, quel effet de vérité ça produit, indépendamment de la véracité de ses propos. Son registre est celui de l'apparence, qu'il s'applique à faire briller d'un éclat irrésistible. Persuadé que la vérité doit rester au fond du puits où elle s'est retirée, le pervers travaille à bâtir une réalité de remplacement, qu'il impose comme la seule nécessaire. Son pouvoir de séduction, il le tire de son habileté à convaincre, de l'admiration que ne manquent jamais de provoquer ses constantes performances. Tant qu'il est capable de soutenir l'illusion qu'il a créée, il est protégé contre l'obligation de justifier son système et d'en vérifier l'authenticité. Faux-monnayeur peut-être, mais pour lui-même, faisant circuler les valeurs qu'il fabrique pour son plaisir, il n'est pas responsable du leurre qu'elles représentent, puisque dans son jeu à lui elles sont les seules garanties possibles. Il y a parfois des situations d'urgence qui demandent un complet renversement des règles établies, dans lesquelles il faut inventer un nouveau type de fonctionnement

14 Jean-Paul Valabrega, dans la discussion qui suit le travail de Guy Rosolato, "Etudes des perversions sexuelles à partir du fétichisme", dans Piera AulagnierSpairani et autres, Le désir et la perversion, coll. "Points", Paris, Seuil, 1967. p. 43. 
qui permette d'éviter des complications pénibles, voire des catastrophes irréparables. Le pervers est toujours en situation d'urgence: devant l'épreuve de vérité que constitue la castration, il a choisi d'en taire l'horreur, entourant son silence de mirages et de fables qui le détournent de ses angoisses et de ses peurs. Par cette conjuration de la réalité, il espère davantage que de simplement brouiller le sens de la castration; il veut rendre le sens sans effet, le décrocher de ses assises, le faire dériver jusqu'à se perdre dans le nonsens. Il fait le pari de la réversibilité des choses: pour peu qu'on se donne la peine de jouer avec les apparences, de prendre les signes comme des outils de séduction, on s'aperçoit vite que l'absolu de vérité n'existe pas, puisque tout sens qui émerge commence aussitôt à s'engloutir dans son contraire. La seule transparence qui résiste à cette circulation insensée des formes, c'est celle du kaléidoscope, une transparence qui se brise dès qu'on essaie de la toucher.

\section{L'homme au sabre}

L'homme au sabre a rejoint Jean-Jacques, les vieilles femmes armées d'un balai aussi. Le danger est partout. Toutes les avenues sont bouchées:

L'homme au sabre en me prenant par le bras me demanda rudement ce que je faisois-là. On conçoit que ma réponse n'étoit pas prette. Je me remis, cependant, et m'évertuant dans ce moment critique je tirai de ma tête un expédient romanesque qui me réussit. Je lui dis d'un ton suppliant d'avoir pitié de mon âge et de mon état; que j'étois un jeune étranger de grande naissance dont le cerveau s'étoit dérangé; que je m'étois échapé de la maison paternelle parce qu'on vouloit m'enfermer, que j'étois perdu s'il me faisoit connoître; mais que s'il vouloit bien me laisser aller je pourrois peutêtre un jour reconnoitre cette grâce. Contre toute attente, mon discours et mon air firent effet. L'homme terrible en fut touché, et après une réprimande assez courte, il me laissa doucement aller sans me questionner davantage. ${ }^{15}$

Jean-Jacques a une réaction magnifique. Il s'arrange pour éviter la castration au nom même de la castration. Pourquoi, en effet, le menacer d'un châtiment qui n'ajouterait rien

Jean-Jacques Rousseau, Les confessions, 3, t. I, p. 90. 
56

à sa misère présente, dont il subit déjà toutes les contraintes? Qu'il soit * de grande naissance *, c'est certain mais cela compte-t-il puisqu'il a tout perdu, ou presque? Son père finira bien par retrouver sa trace et il imposera sa loi. Pourquoi un inconnu voudrait-il se substituer à l'autorité parentale et faire ce que, de toute manière, on fera un jour: mettre le pauvre garçon en face de sa folie? Ne faut-il pas avoir pitié? Jean-Jacques a gagné. L'homme au sabre est séduit et il devient complice. Et complice d'un mensonge! La tromperie est si énorme que Jean-Jacques lui-même s'étonne de la crédulité de l'autre et mesure toute l'impudence de son discours. La réussite n'en est que plus triomphante. JeanJacques sait à présent qu'on peut risquer son être sur un paraître, fût-il hypocrite, et que les apparences, surtout si elles mentent, sont souvent à l'origine même du sens.

Que sont devenues les femmes dans toute cette histoire? Elles traînent en arrière, sans doute pas très loin, miraculeusement reléguées à un rôle de second plan. Comme si elles n'avaient été qu'un prétexte nécessaire à l'enclenchement d'un drame où, finalement, elles se révéleraient de simples figurantes:

A l'air dont la jeune et les vieilles me virent partir, je jugeai que I'homme que j'avois tant craint m'étoit fort utile, et qu'avec elles seules je n'en aurois pas été quitte à si bon marché. Je les entendis murmurer je ne sais quoi dont je ne me souciois guère; car pourvu que le sabre et l'homme ne s'en mêlassent pas, j'étois bien sûr, leste et vigoureux comme j'étois, de me délivrer et de leurs tricots et d'elles. ${ }^{16}$

Il y a ici quelque chose qui ressemble à une échappatoire. Le raisonnement, en effet, débouche sur un bien curieux déni, celui de la castration apportée par la femme. Tout semble s'organiser pour éloigner la menace réelle que constitue le monde féminin, comme si la castration n'était autorisée à venir que de l'homme, comme si elle invalidait alors complètement l'agressivité de la femme. C'est du moins ce que le texte suggère, mais après une sorte d'hésitation, avec dans l'air une appréhension diffuse, mal évaluée. Jean-Jacques sait que la violence des femmes ne désarme pas facilement, mais on dirait qu'il ne la craint plus quand elle se déploie dans l'ombre d'un pouvoir phallique, tant qu'elle est vécue 
par mode de comparaison. Elle se trouve alors comme retenue dans un espace d'emprunt, qui désamorce sa charge et la rend inoffensive. À côté du sabre, le balai n'est qu'une caricature rassurante, devant laquelle Jean-Jacques redevient tout à coup «leste et vigoureux*. Il sait déjà pourtant qu'il s'en fallait de peu pour que les choses se passent autrement, pour que le paysage soit brouillé et que s'impose l'évidence de la catastrophe.

Ce qui émerge ici, c'est l'attitude fondamentale de JeanJacques devant l'éventualité de la castration. C'est son horreur de découvrir l'opacité du monde, ce manque de limpidité, cette absence de connivence. C'est son angoisse devant le rejet dans la solitude, dans le silence des incompréhensions, dans l'incertitude des différences. Mais c'est aussi, et en même temps, un espoir insencé que cette immense hésitation du réel demeure, que cette ambiguilé ne soit pas levée, pour que ne soit pas imposé définitivement le choc de l'incomplétude. Quand on a compris qu'il existe une vérité en dehors de soi, c'est-à-dire une possibilité de mensonge, il faut se garder d'une imprudente curiosité, au bout de laquelle attend peut-être une insurmontable impuissance.

Une fois ainsi désavouée l'évidence des certitudes, et reportés indéfiniment les effets de la contradiction, qu'est-ce qui saurait encore empêcher l'interprétation - cette lecture différante qu'un sujet fait d'une parole - de s'ouvrir à du sens jusque là retenu dans les plis de la métaphore, gardé en réserve pour que soient aussi signalées les ellipses de l'imaginaire? Et à ceux qui voudraient comprendre cette fonction de liberté de l'interprétation comme un encouragement à lire n'importe quoi dans un texte, je suggère de reprendre inlassablement l'exercice qui vient d'être fait: ils finiront bien par voir qu'il n'y a pas de lumière sans ombre, que les plus éclatantes révélations se font dans le silence d'un secret. 